\title{
EFEITO DA LAMINAÇÃO A QUENTE E A MORNO NA MICROESTRUTURA, DUREZA E TEXTURA DE UM AÇO MÉDIO MANGANÊS*
}

\section{Resumo}

Aline Silva Magalhães ${ }^{1}$ lane Dutra Moutinho² Indiana Rosa Oliveira ${ }^{2}$ Aline Oliveira Vasconcelos Ferreira ${ }^{2}$ Davi Silva Alves ${ }^{2}$ Carolina Arriel Pedroso Dias ${ }^{2}$ Graziele Gianini Braga Maria ${ }^{3}$ Dagoberto Brandão Santos ${ }^{4}$

Os aços com médio teor manganês são uma classe da terceira geração de aços avançados de alta resistência (AHSS), combinam propriedades mecânicas como ductilidade e resistência mecânica e são assistidos pelo efeito TRIP, características determinantes para sua aplicação na indústria automobilística. Convencionalmente, tais ligas são produzidas por meio de laminação a quente e a frio, seguidas de recozimento intercrítico. No entanto, o trabalho a morno têm ganhado interesse com o intuito de reduzir custos e tempo operacionais. O objetivo deste trabalho foi comparar a influência da laminação a quente e a morno por meio de análises de microscopia óptica, eletrônica de varredura, difração de raios $\mathrm{x}$, microdureza Vickers e técnicas de EBSD em um aço 8Mn$0,08 \mathrm{C}$. Observou-se uma microestrutura mais refinada e deformada no material laminado a morno com um maior percentual de austenita retida, o que derivou uma menor dureza. $\mathrm{Na}$ amostra laminada a quente, o valor médio de dureza evidenciou uma microestrutura martensítica de baixo teor de carbono. Em relação à textura verificou-se uma maior intensidade de componentes da fibra a na amostra laminada a morno devido a um maior grau de encruamento neste processamento termomecânico.

Palavras-chave: Aço médio manganês; Efeito TRIP; Laminação a quente; Laminação a morno.

\section{HOT AND WARM ROLLING EFFECT IN MICROSTRUCTURE, HARDNESS AND TEXTURE OF A MEDIUM MANGANESE STEEL}

\section{Abstract}

Medium manganese steels are a grade of the third generation of advanced high strength steels (AHSS) that combine mechanical properties such as ductility and tensile strength, determining characteristics for application in the automotive industry. Conventionally, these steels are subjected to a hot and cold rolling followed by intercritical annealing. However, the warm rolling acquire interest in order to reduce costs and operating time. The aim of this study it is to compare the influence of hot and warm rolling by optical and scanning electron microscopy analysis, X-ray diffraction, Vickers hardness and EBSD in a $8 \mathrm{Mn}-0,08 \mathrm{C}$ steel. A refined and deformed microstructure in warm-rolled sample was observed, with a higher volume fraction of retained austenite, which derived a lower hardness. In the hot-rolled sample, the average hardness value revealed a martensite with a low carbon content microstructure. The texture shows a greater intensity of the a fiber components in warm rolled to warm due to higher strain in this thermomechanical processing.

Keywords: Medium manganese steel; TRIP effect; Hot rolling; Warm rolling.

1 Eng. Materiais, ME, Professora do Departamento de Engenharia de Materiais do CEFET-MG, Doutoranda no Programa de Pós-Graduação em Engenharia Metalúrgica, Materiais e de Minas da UFMG, Belo Horizonte, MG, Brasil.

2 Graduando Eng. Metalúrgica, Iniciação científica no Departamento de Engenharia Metalúrgica, UFMG, Belo Horizonte, MG, Brasil.

3 Eng. Materiais, ME, Doutoranda no Programa de Pós-Graduação em Engenharia Metalúrgica, Materiais e de Minas, UFMG, Belo Horizonte, MG, Brasil.

4 Eng. Metalurgista, Dr., Professor titular, Departamento de engenharia Metalúrgica e de Materiais, UFMG, Belo Horizonte, MG, Brasil. 


\section{INTRODUÇÃO}

A indústria automobilística tem exigido, ao longo de décadas, o desenvolvimento de novos materiais com menor peso e maior resistência mecânica para atender uma demanda mundial de redução do consumo de combustíveis, de maior absorção de energia ao impacto, a fim de garantir segurança aos passageiros, e melhor conformabilidade, no intuito de favorecer os processos de estampagem.

Os aços avançados de alta resistência (AHSS) desenvolvidos para atender à essas exigências, são caracterizados, de maneira geral, por uma microestrutura multiconstituída, podendo conter ferrita, martensita, bainita e austenita retida, com o propósito de otimizar a combinação de resistência mecânica e ductilidade [1].

Os aços com médio teor manganês compõem a terceira geração dos aços avançados de alta resistência, apresentam um teor de manganês entre 3 a 12\% [2], exibem boa ductilidade, elevada resistência mecânica e maior viabilidade econômica devido a um menor teor de ligas, quando comparados aos AHSS da segunda geração.

A microestrutura desses aços, geralmente é composta de martensita, ferrita e austenita retida, esta última sendo responsável pelo efeito TRIP (transformação induzida por deformação). Esse fenômeno ocorre durante a deformação plástica, quando a austenita retida metaestável se transforma em martensita, promovendo um aumento significativo na resistência mecânica do material [3]. A ductilidade apresentada pelos aços médio manganês provém da composição microestrutural contendo ferrita, macia e dúctil, e austenita retida, enquanto a alta resistência resulta da martensita gerada no efeito TRIP [4].

Convencionalmente, esses aços são produzidos a partir de um processamento envolvendo laminação a quente com posterior laminação a frio e recozimento intercrítico. No entanto, a laminação a morno têm ganhado interesse com a finalidade de reduzir custos e tempo operacional. Enquanto a laminação a morno pode ser feita na faixa de $650^{\circ} \mathrm{C}$ a $1000^{\circ} \mathrm{C}$, a laminação a quente demanda temperaturas na faixa de $850^{\circ} \mathrm{C}$ a $1250^{\circ} \mathrm{C}$, de acordo com a composição química do aço [5].

Neste trabalho, os efeitos da laminação a quente e a morno na microestrutura, na textura e na dureza um aço médio manganês são comparados por meio das técnicas de microscopia óptica e eletrônica de varredura, difração de raios X, difração de elétrons retroespalhados e microdureza Vickers.

\section{MATERIAIS E MÉTODOS}

O material utilizado para o desenvolvimento deste estudo consiste em blocos de aço médio manganês obtidos por meio de fusão em forno elétrico a indução convencional. A composição química, exibida na Tabela 1, é resultado da análise por espectrometria de emissão óptica, realizada em um equipamento Shimadzu Modelo Foundry Master Xpert.

Tabela 1. Composição química (\% em peso) do aço médio manganês

\begin{tabular}{cc}
\hline Elemento & Teor (\%) \\
\hline $\mathrm{Fe}$ & $91,50 \%$ \\
\hline $\mathrm{C}$ & $0,08 \%$ \\
\hline $\mathrm{Mn}$ & $8,05 \%$ \\
\hline $\mathrm{Si}$ & $0,14 \%$ \\
\hline $\mathrm{Al}$ & $0,02 \%$ \\
\hline
\end{tabular}


$\mathrm{O}$ aço foi aquecido a $1100^{\circ} \mathrm{C}$ por $2 \mathrm{~h}$, com o objetivo de homogeneizar a microestrutura desenvolvida durante a solidificação. Posteriormente, os blocos foram divididos em duas séries de processamento termomecânico: laminação a quente e a morno, respectivamente, realizadas em um laminador de laboratório Fröhling.

A laminação a quente foi conduzida a $1100^{\circ} \mathrm{C}$ em quatro passes, com encharque inicial de 30 min e aquecimento na mesma temperatura de 10 min entre passes. Posteriormente, a amostra foi resfriada ao ar, resultando na espessura de $8 \mathrm{~mm}$, com $\varepsilon=0,62$. O processo de laminação a morno foi realizado a $630^{\circ} \mathrm{C}$ durante $30 \mathrm{~min}$, incialmente e 15 min de aquecimento a $630^{\circ} \mathrm{C}$ entre cada um dos onze passes, obtendo-se a espessura final de $8,5 \mathrm{~mm} \operatorname{com} \varepsilon=0,72$, seguido de resfriamento ao ar. A microestrutura foi caracterizada por meio das técnicas de microscopia óptica e eletrônica de varredura. As amostras foram preparadas de acordo com procedimento metalográfico convencional, envolvendo seccionamento, embutimento a quente, lixamento em lixas \#100, \#240, \# 320, \#400, \#600, \#1000 e \#2400, e polimento com pasta de diamante de $9 \mu \mathrm{m}, 3 \mu \mathrm{m}, 1 \mu \mathrm{m}$ e solução OPS durante $3 \mathrm{~min}$. Posteriormente, as amostras foram submetidas a ataque químico com o reativo nital $2 \%$.

Por meio da análise por difração de raios $X$, utilizando um difratômetro Panalitical Modelo Empyrean com condições operacionais de ângulo de $30^{\circ}$ a $120^{\circ}$ e passo de 0,02 , foi possível determinar as fases presentes (com mais de $3 \%$ em volume da amostra) e suas relativas quantidades pelo método de integração dos picos de difração, com o auxílio do software Origin ${ }^{\mathrm{TM}}$. Para a observação da microtextura, foi feita análise por EBSD (difração por elétrons retroespalhados) após procedimento metalográfico convencional finalizado com polimento na polimetriz automática MiniMet em sílica coloidal por uma hora. Finalmente, foram realizadas medições de microdureza Vickers com carga de $2,94 \mathrm{~N}$ por $10 \mathrm{~s}$ em 20 pontos distintos na amostra.

\section{RESULTADOS E DISCUSSÃO}

As microestruturas das amostras laminadas a quente e a morno analisadas por meio de microscopia ótica e eletrônica de varredura são exibidas nas Figuras 1 e 2 em aumentos de 500X e 1000X. No material submetido ao processo de laminação a quente (Fig1 a,c e Fig2 a,c,e), observa-se uma estrutura constituída por ferrita e martensita, esta caraterizada por sua morfologia acentuadamente acicular.

Nota-se que a partir da laminação a morno obteve-se uma microestrutura mais refinada (Fig1 b,d) e deformada (Fig2 b,d,f), resultante da menor temperatura de trabalho, consistindo em ferrita, austenita retida e pacotes de martensita. Santos et al. [6] observaram que o aumento da quantidade de deformação final do material submetido à laminação a morno resulta em um refinamento da microestrutura.

Na Figura 3 são mostrados os difratogramas do material e, ao compararmos as duas condições de laminação, observa-se, de maneira qualitativa, que a amostra submetida à laminação a morno apresenta além de picos correspondentes às fases ferrita/martensita $\alpha^{\prime}$, um aumento na quantidade dos picos de austenita $(\gamma)$ e a presença da fase martensita $\varepsilon$, quando comparada com o material conduzido à laminação a quente. Esta condição, por sua vez, exibe picos, em maioria, correspondentes à ferrita/ martensita $\alpha$ ' e pequena quantidade de austenita $(\gamma)$. 


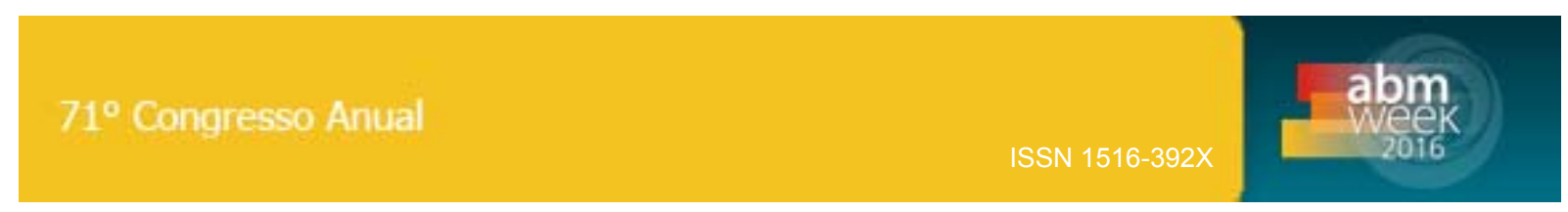

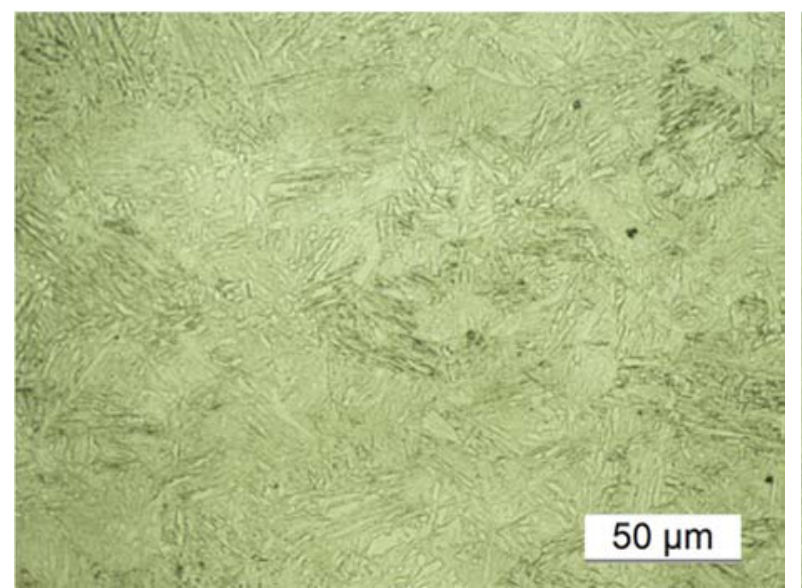

(a) $500 x$

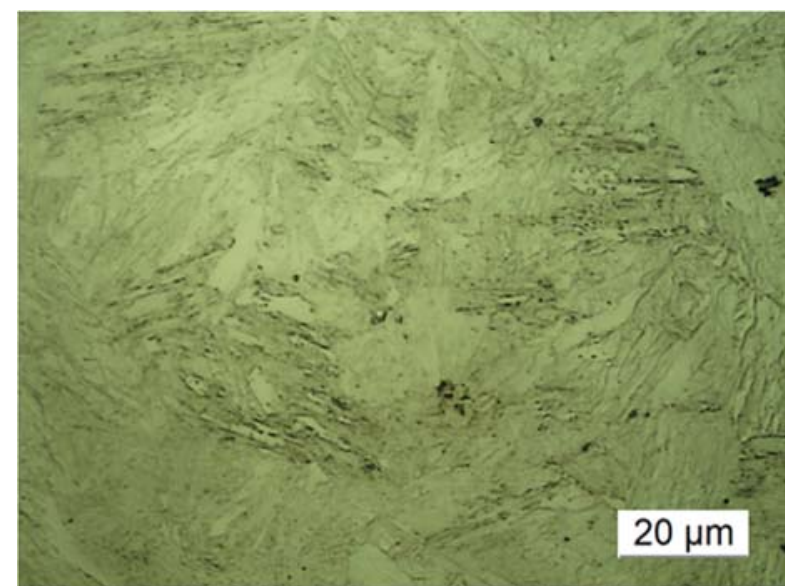

(c) $1000 x$

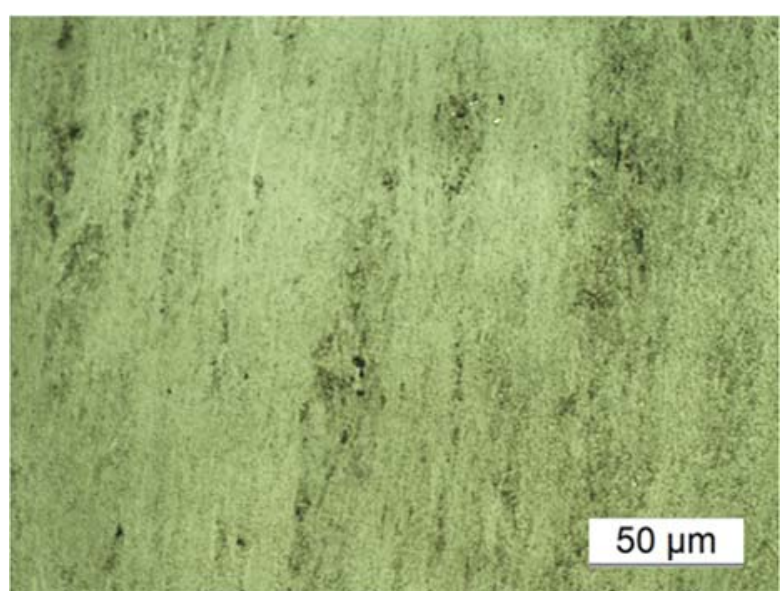

(b) $500 x$

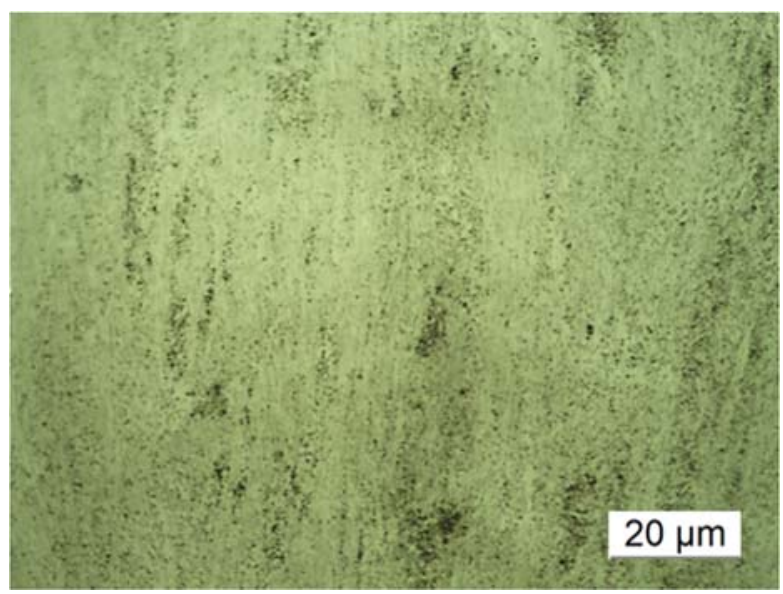

(d) $1000 x$

Figura 1. Microscopias ópticas do material submetido a laminação a quente (a e c) e a morno (b e d) em aumentos distintos.

De forma quantitativa, conforme exibido na Tabela 2, observa-se que há uma maior fração de austenita no material laminado a morno (34\%), o que se deve a uma menor temperatura de trabalho nessa condição, que pode refletir em uma maior estabilidade térmica da austenita, devido a variações menos significativas nas concentrações de carbono e manganês nessa fase [4], quando comparada com o material processado em uma temperatura superior, ou seja, laminado a quente. Em temperaturas mais elevadas, a austenita possui menor estabilidade mecânica, devido à menor concentração de carbono nessas condições, assim, no resfriamento subsequente a austenita se transforma prontamente em ferrita e/ou martensita dependendo da taxa de resfriamento, dificultando sua retenção na microestrutura. Além disso, uma maior densidade de deslocações na austenita do material laminado a morno retém o carbono nessa fase, dado que esse elemento segrega nestas imperfeições [4].

Segundo Aydin et al.[7] e Escobar et al.[8], a ocorrência de martensita $\varepsilon$ está relacionada com a deformação à qual o material laminado a morno foi submetido. $\mathrm{A}$ martensita $\varepsilon$ é considerada uma fase intermediária na transformação de fases da austenita $\gamma$ (CFC) para a martensita $\alpha^{\prime}$, podendo ser escrita como $\gamma$ (CFC) - martensita $\varepsilon(\mathrm{HC})$ - martensita $\alpha^{\prime}[8]$. 


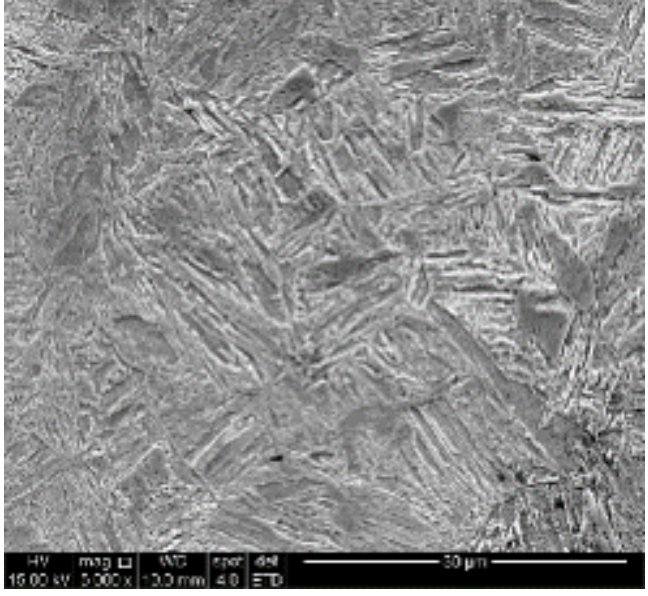

(a) $5000 x$

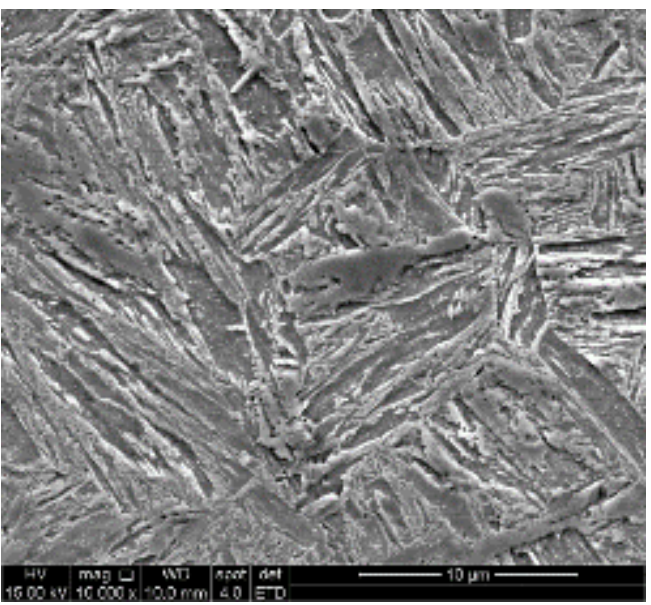

(c) $10000 x$

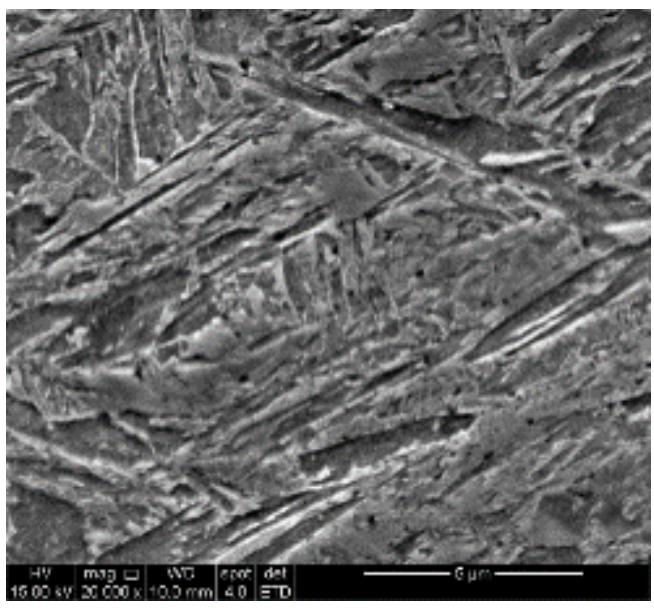

(e) $20000 x$

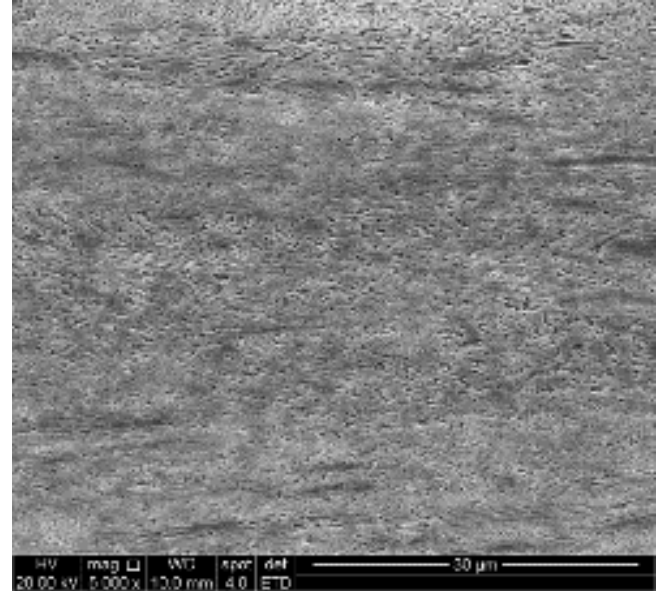

(b) $5000 x$

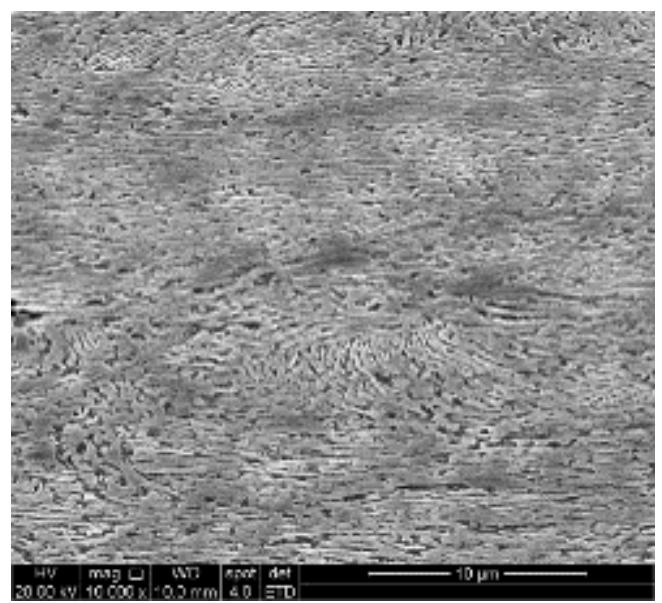

(d) $10000 x$

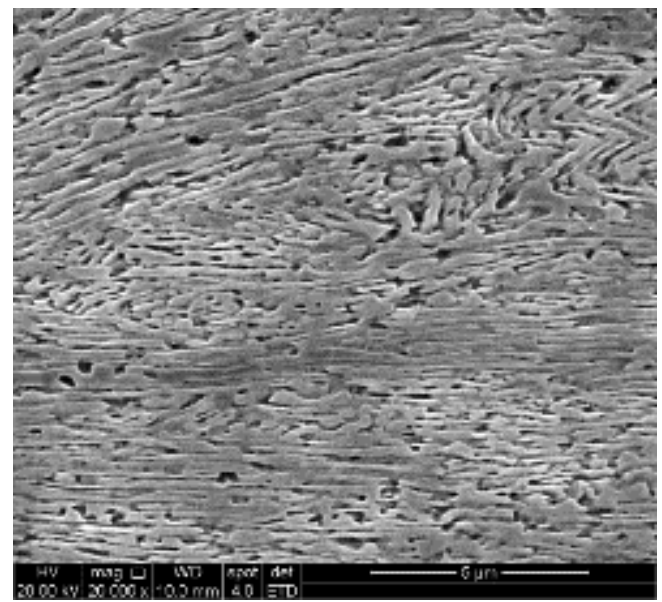

(f) $20000 x$

Figura 2. Microscopia eletrônica de varredura das amostras $(a, c, e)$ laminada a quente e (b,d,f) laminada a morno em aumentos distintos. 


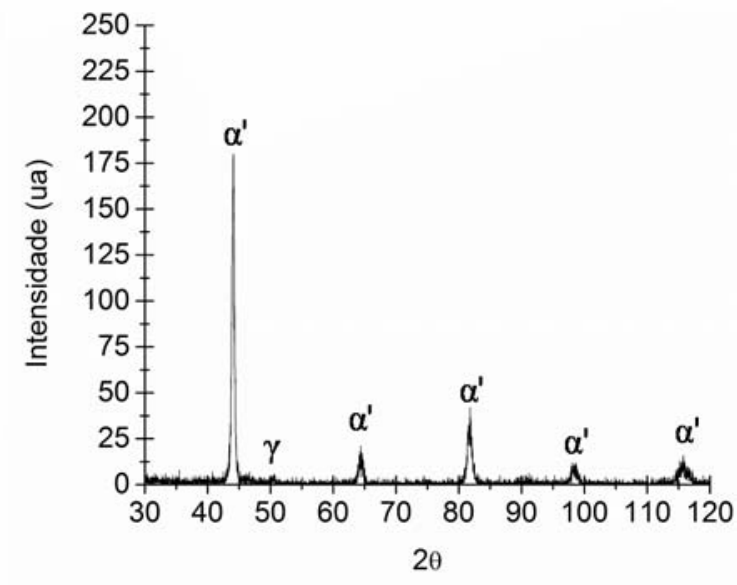

(a)

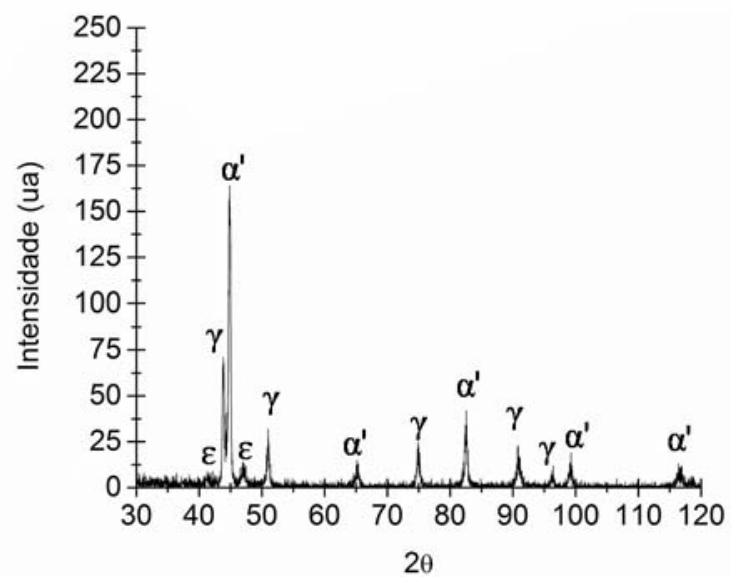

(b)

Figura 3. Difratogramas do material laminado (a) a quente e (b) a morno.

Tabela 2. Análise quantitativa das fases por difração de raios $X$

\begin{tabular}{lccc}
\hline Condição & \% Martensita $\boldsymbol{\alpha}$ & \% Martensita $\varepsilon$ & $\%$ Austenita \\
\hline Laminado a quente & $99,0 \%$ & - & $1,0 \%$ \\
\hline Laminado a morno & $62,0 \%$ & $4,0 \%$ & $34,0 \%$ \\
\hline
\end{tabular}

Os valores de microdureza Vickers para o material nas condições experimentais são mostrados na Tabela 3, juntamente com os desvios-padrão das medidas. O material laminado a quente apresenta um valor médio de dureza característico de uma martensita de baixo teor de carbono. Observa-se uma dureza superior do material submetido a laminação a quente em comparação com o laminado a morno, o que, supõe-se estar relacionado com uma menor quantidade de austenita retida na estrutura, corroborada pelos resultados quantitativos de difração de raios $\mathrm{X}$, em comparação com a fração volumétrica de martensita, resultando assim em um maior valor de dureza para essa condição.

Tabela 3. Dureza das amostras laminadas a quente e a morno

\begin{tabular}{lc}
\hline Condição & Dureza Vickers \\
\hline Laminado a quente & $413 \pm 11 \mathrm{HV}$ \\
\hline Laminado a morno & $314 \pm 10 \mathrm{HV}$ \\
\hline
\end{tabular}

As figuras de polo inversas (IPF) da textura do material laminado a quente e a morno, obtidas pelo software $\mathrm{OIM}^{\mathrm{TM}}$ são exibidas na Figura 4. Na amostra conduzida à laminação a quente, nota-se que não é possível observar uma orientação preferencial, característica oriunda do fenômeno de recristalização. Verifica-se um baixo índice de indexação dos dados devido à maior concentração de defeitos cristalinos nos grãos da amostra submetida à laminação a morno. Este comportamento se relaciona com uma elevada densidade de defeitos na estrutura como lacunas e deslocações, relacionados à uma maior quantidade de deformação à qual o material é submetido em temperaturas mais baixas, quando comparado à laminação a quente. 


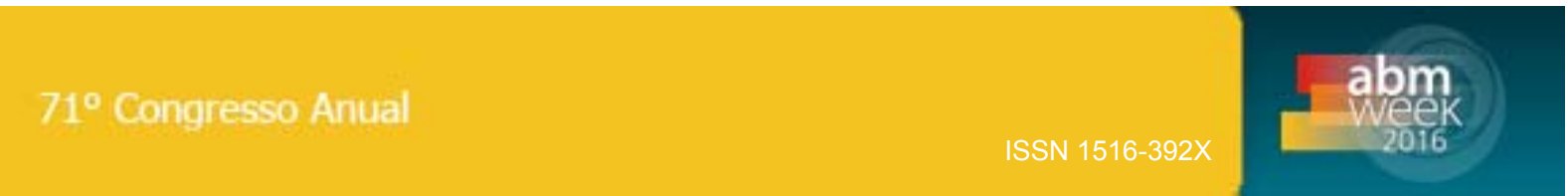

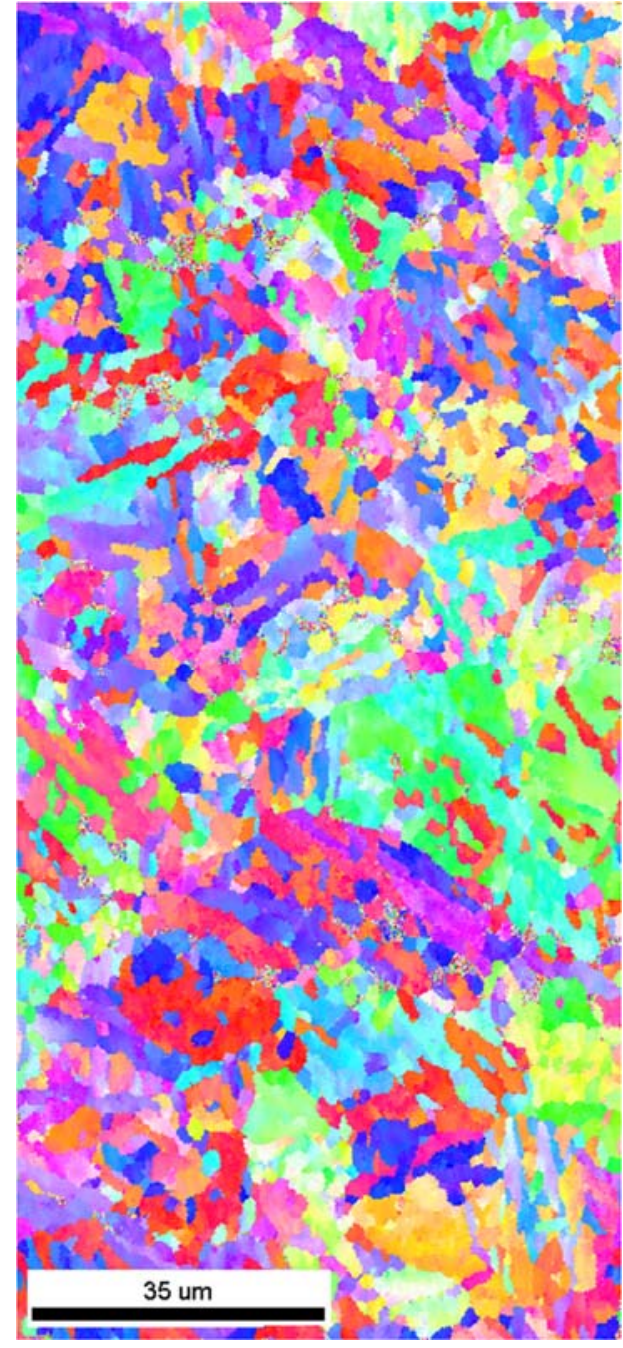

(a)

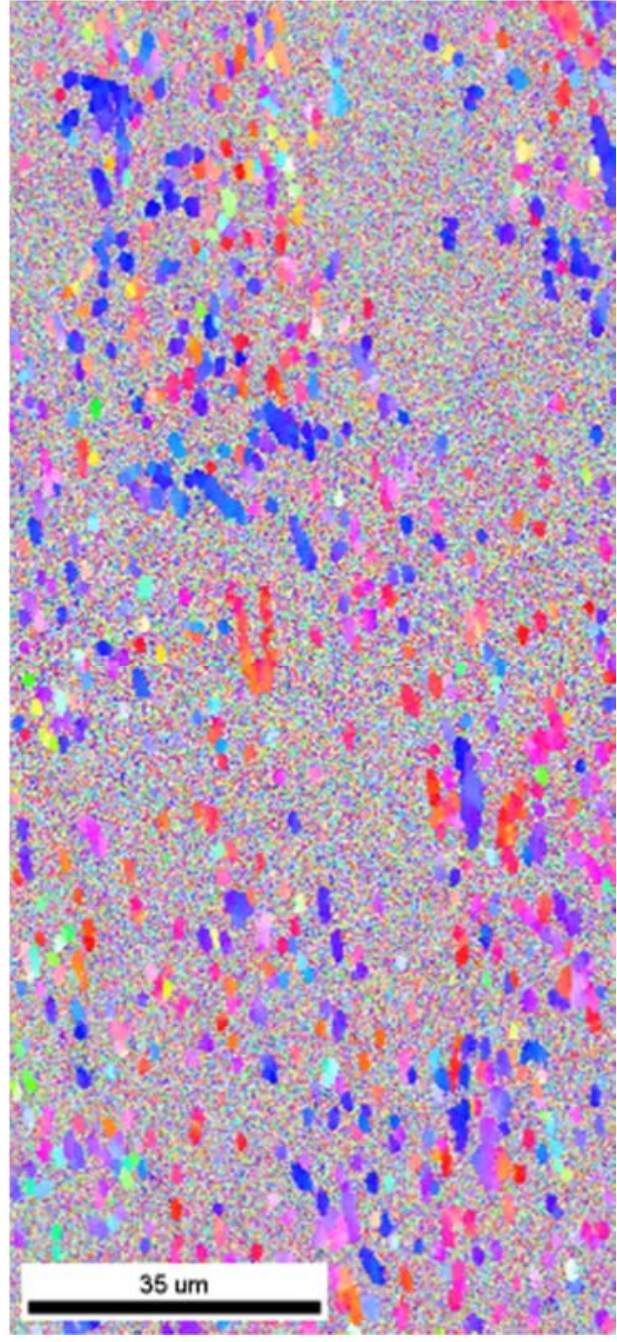

(b)

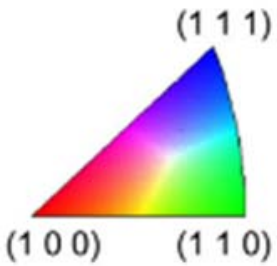

Figura 4. IPF (Inverse Pole Figure) das amostras (a) laminada a quente e (b) laminada a morno.

A Figura 5 exibe as texturas do material descritas pelas funções de distribuição de orientação (ODF) para $\varphi 2=45^{\circ}$ para a martensita $\left(\alpha^{\circ}\right)$. A textura do material laminado a quente apresenta retenção da componente fibra $\alpha$, mesmo que a tendência indique que esta seja consumida durante a recristalização [9].

A maior intensidade de textura está relacionada à componente (001)[-1-20] e a componente cubo girado (001)[2-30]. Observa-se ainda a presença da componente goss girado (110)[1-10] com baixa intensidade.

No material laminado a morno nota-se a maior intensidade da componente $\mathrm{Cu}$ transformado (-1-13)[-1-10] pertencente à fibra $\alpha$, característica do material deformado. A componente de textura cubo girado (001)[1-10] apresentou baixa intensidade.

De maneira geral, não foram observadas alterações significativas na intensidade da fibra $\gamma$ com a diminuição da temperatura, e esta apresentou baixas intensidades de seus componentes em ambas as temperaturas de laminação. A principal componente da fibra $\gamma$ (111)[0-11] ocorre tanto no material laminado a quente quanto a morno. Escobar et al. [8] ao estudar a textura da martensita em um aço $17 \mathrm{Mn}-0,06 \mathrm{C}$ observaram duas componentes da fibra gama: (111)[1-10] e (111)[0-11]. 
Kesten et al. [10] relatam que quanto menor a temperatura final de laminação a quente, ou seja, quanto mais intensa a deformação no campo ferrítico, a fibra alfa é fortalecida, especialmente o componente (100)[011] e menos intensa a fibra gama. Kurc-Lisiecka et al. [11] destacam que a textura da martensita é mais complexa, uma vez que compreende os componentes de textura da martensita originária da austenita recém desenvolvida em um dado estágio de deformação assim como da formada previamente e já deformada.

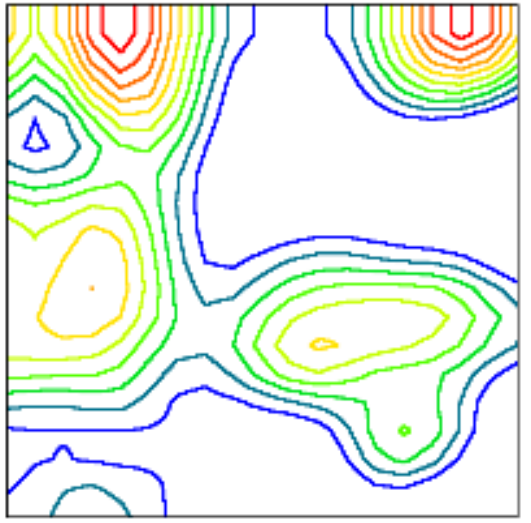

(a)

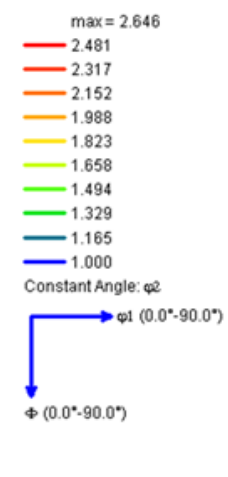

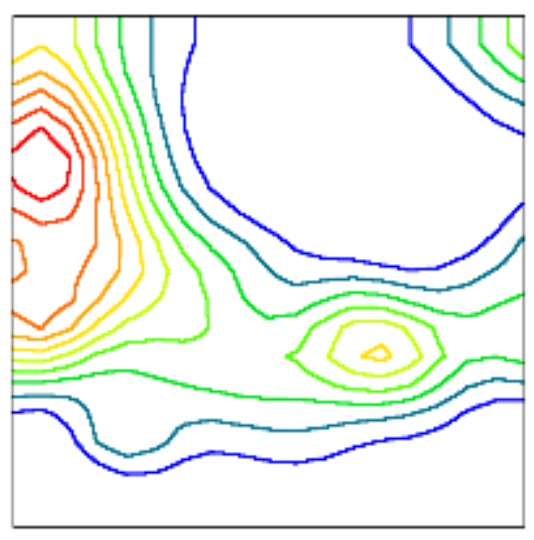

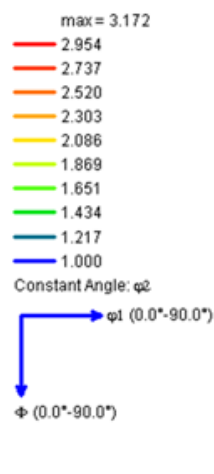

(b)

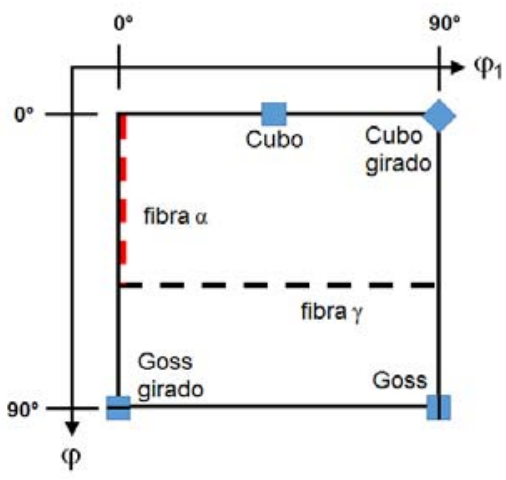

(c)

Figura 6. ODF (Orientation Distribution Function) para $\varphi 2=45^{\circ}$ das amostras (a) laminada a quente e (b) laminada a morno e (c) localização das fibras e orientações características.

\section{CONCLUSÕES}

Comparativamente, observou-se que a laminação a morno resultou em uma microestrutura mais refinada e deformada em relação à laminação a quente, com uma maior fração volumétrica de austenita, o que derivou uma menor dureza. Para o material laminado a quente, o valor médio de dureza evidenciou uma microestrutura composta de martensita com baixo teor de carbono. Em relação à textura, observouse uma maior intensidade de componentes da fibra $\alpha$ na amostra laminada a morno, devido a uma maior deformação atuante neste processamento termomecânico. 


\section{Agradecimentos}

Os autores agradecem às agências de fomento CAPES-PROEX (Coordenação de Aperfeiçoamento Pessoal de Nível Superior) e CNPq pela concessão das bolsas de pesquisa e apoio financeiro.

\section{REFERÊNCIAS}

1 Tamarelli, C.M. The Evolving Use of Advanced High-Strength Steels for Automotive Applications. Steel Market Development Institute, Materials Science and Engineering. 2011. [acesso em 31 mar. 2016]. Disponível em: http://www.autosteel.org.

2 Sun, B.; Aydin, H.; Fazeli, F.; Yue, Stephen. Microstructure evolution of a medium manganese steel during thermomechanical processing. Metallurgical and Materials Transactions A. 2016; 47, 1782-1791.

3 Xu, H.F.; Zhao, J.; Cao, W.Q.; Shi, J.; Wang, C.Y.; Wang, C.; Li, J.; Dong, H. Heat treatment effects on the microstructure and mechanical properties of a medium manganese steel $(0,2 \mathrm{C}-5 \mathrm{Mn})$. Materials Science and Engineering A. 2012; 532, 435442.

4 Zhao, X.; Shen, Y.; Qiu, L.; Liu, Y.; Sun, X.; Zuo, L. Effects of intercritical annealing temperature on mechanical properties of Fe-7.9Mn-0.14Si-0.05Al-0.07C steel. Materials, 2014; 7, 7891-7906.

5 Bruna, R.G. Efeitos dos laminados a quente e a morno na microestrutura, textura e propriedades de aços baixo carbono. Rem: Revista Escola de Minas, 2011; 64, 57-62.

6 Santos, D.B.; Bruzszek, R.K.; Rodrigues, P.C.M.; Pereloma, E.V. Formation of ultra-fine ferrite microstructure in warm rolled and annealed C-Mn steel. Materials Science Engineering A, 2003; 346, 189-195.

7 Aydin, H.; Essadiqi, E.; Jung, H.; Yue, Stephen. Development of 3rd generation AHSS with medium Mn content alloying compositions. Materials Science Engineering A, 2013; 564, 501-508.

8 Escobar, D.P.; Dafé, S.S.F.; Santos, D.B. Martensite reversion and texture formation in $17 \mathrm{Mn}-0,06 \mathrm{C}$ TRIP/TWIP steel after hot cold rolling and annealing. Journal of Materials Research and Technology. 2015; 4, 162-170.

9 Viana, C.S.C.; Paula, A.S. Texturas de deformação. Instituto Militar de Engenharia. 2002.

10 Kestens, L.; Jonas, J.J.; Van Houtte, P.; Aernoudt, E. Orientation selective recrystallization of nonoriented electrical steels. Metallurgical and Materials Transactions A. 1996; 27, 2347-2358.

11 Kurc-Lisiecka, A.; Ozgowicz, W.; Ratuszek, W.; Chruściel, K. Texture and structure evolution during cold rolling of austenitic stainless steel. Journal of Achievements in Materials and Manufacturing Engineering. 2012, 52, 22-30. 